\title{
EDITORIAL
}

\section{Light technology potential in reducing secondary infections in the dental office.}

\author{
Edwin Sever Bechir ${ }^{1}$ \\ ${ }^{1}$ George Emil Palade University of Medicine, Pharmacy, Science, and Technology of Târgu-Mureș, Târgu Mureș, \\ Romania
}

In the current context of the exponential spread of the new coronavirus (SARS-CoV-2) and its associated disease globally, and also in Romania, we are faced with a growing concern in the way it affects us both in terms of public health as well as personally in the activity we carry out every day in our field of work.

Unfortunately, it seems that this pandemic will not end shortly, implicating that we will have to adapt to the new conditions and consider the current protocols as rules that will need to be applied from now on for an extended period of time. During this timeframe, a significant number of people will probably avoid dental treatments (excepting dental emergencies) both because of the way this pandemic affects us financially, but before all else for psychological reasons: it will be difficult for the patients to overcome their fear of falling victim to this novel virus. For many of these, the dental office is a potential source of infection, primarily considering that the person most exposed to this risk is the dentist himself [1].

Studies showed abundant presence of SARS-CoV-2 in the nasopharyngeal and salivary secretions of affected patients, and that it predominantly spreads through respiratory droplets [2]. The risk of transmission amongst medical workers has been documented and the risk of infection is higher in the case of dentists, dental assistants, oral and maxillofacial surgeons (with a risk of exposure within 95\% to $100 \%$ ) because of their constant work in close vicinity of aerosols and saliva droplets generated by the various procedures performed on patients.

This is the reason why dental office managers and especially doctors must constantly adjust and improve operating protocols to protect both the team members as well as the patients [3,4]. After performing dental procedures, the doctor often removes his mask to talk to the patient. Removing this protective barrier may prove to be a mistake because aerosols can remain in the air for up to 30 minutes thus increasing the potential of contact with contaminated material [5].

The current guidelines for dental practice are constantly evolving and mainly target a specific purpose, namely the diminution of the transmission risk of viruses and bacteria both amidst medical staff and patients as well as from one patient to another.

In addition to the use of standard precautions such as the use of protective barriers (masks, caps, gowns, visors, gloves, etc.), the disinfection of instruments and equipment, the use of substances that can reduce coronavirus infectivity (e.g. hydrogen peroxide in a concentration of $0.5 \%$ has proven effective when used in oral rinses for 1 minute), which represent the fundamental actions to minimize cross infections in the dental office, the use of lasers can play a major role in diminishing the risk of SARS-CoV-2 transmission by the very fact that they are not included in the list of sources that create aerosols $[6,7]$.

The Centers for Disease Control and Prevention concluded that when practicing dentistry the risk of SARS-CoV-2 transmission during aerosol generating dental procedures cannot be eliminated, and recommended avoiding these procedures whenever possible [8].

According to Zemouri $\mathrm{C}$, de Soet $\mathrm{H}$, Crielaard W, Laheij A the aerosol sources in dental clinics are represented by: ultrasonic scalers, high speed hand pieces and the air and 
water syringe from the dental unit [9]. The water spray is an important factor in creating aerosols in the dental office, aerosols that can cause the transmission of pathogenic viruses.

The evolution of dental lasers currently allows us to perform a large number of procedures, including cavity preparation, periodontal procedures, restorations, crown lengthenings, frenectomies and many others while reducing the aerosols produced by conventional instruments.

Lasers have the advantage that they can be used for both soft and hard tissues either by using water and air spray or by stopping the water and air spray altogether. When working without water and air spray, the risks caused by aerosols in the spread of bacteria and viruses, including SARS-CoV-2, are considerably reduced while using lasers compared to conventional rotary instruments.

The mechanical way of cutting with the high speed drills causes a spread of dental tissue residue outside the oral cavity that can contain bacteria and viruses with harmful potential. Lasers, on the other hand, perform the ablation of small areas of dental tissue, causing a reduced spread of tissue debris outside the oral cavity. At the same time, these lasers have an antimicrobial capacity, thus significantly reducing the risk of infection. In this context, lasers should become an essential tool in our daily practice considering they allow a way to minimize the production of aerosols and water and saliva droplets therefore reducing the risk of SARS-CoV-2 infection [7].

The constant evolution in light technology proved the therapeutic effects of different light spectra, particularly violet/blue, red and near infrared light. Studies have shown that light in the range of $400-470 \mathrm{~nm}$ (blue light) has an antimicrobial effect against a number of bacteria and it can potentially reduce bacterial suprainfections associated with SARS-CoV-2 and other coronavirus infections. Additionally, wavelengths in the approximate range of 600 $700 \mathrm{~nm}$ and 700-1000 nm (red and near infrared light respectively), have the ability to reduce pulmonary inflammation and fibrosis, and therefore acute respiratory distress syndrome, which represents a leading cause of death in the existing SARS-CoV-2 pandemic. Also, studies provide convincing grounds to investigate the promising effects of several spectra of light in decreasing opportunistic bacterial infections associated with this condition, and their potential use in suppressing SARS-CoV-2 and other viral infections [10].

Lastly, given the existing context, lasers should be considered an option that is safer than the high speed handpieces for both dentists and their patients, offering them state-of-the-art treatments, in a considerably safer setting, with fewer risks and a greater peace of mind.

Conflict of interest: None to declare.

\section{References}

1. Bizzoca ME, Campisi G, Lo Muzio L. Covid-19 Pandemic: What Changes for Dentists and Oral Medicine Experts? A Narrative Review and Novel Approaches to Infection Containment. Int. J. Environmental Research and Public Health. 2020;17(11):3793.

2. Ather A, Patel B, Ruparel NB, Diogenes A. Coronavirus Disease 19 (COVID-19): Implications for Clinical Dental Care. J Endod. 2020;46(5):584595.

3. Meng L, Hua F, Bian Z. Coronavirus disease 2019 (COVID-19):Emerging and future challenges for dental and oral medicine. J Dent Res. 2020;99(5):481-7.

4. Giudice A, Bennardo F, Antonelli A, Barone S, Fortunato L. COVID-19 is a New Challenge for Dental Practitioners: Advice on Patients' Management from Prevention of Cross Infections to Telemedicine. The Open Dentistry Journal. 2020;14:298-304.

5. Micik RE, Miller RL, Mazzarella MA, Ryge G. Studies on dental aerobiology. I. Bacterial aerosols generated during dental procedures. J Dent Res. 1969;48(1):49-56.

6. Kampf G, Todt D, Pfaender S, Steinmann E. Persistence of coronaviruses on inanimate surfaces and their inactivation with biocidal agents. J Hosp Infect. 2020;104(3):246-251.

7. The advantages of using the LiteTouch ${ }^{\mathrm{TM}}$ Er:YAG Laser (by Light Instruments Ltd.) during COVID-19 world health threat and after. - https://www.lightinst.com/covid-19/

8. https://www.cdc.gov/coronavirus/2019ncov/hcp/dental-settings.html

9. Zemouri C, de Soet $H$, Crielaard W, Laheij A. A scoping review on bio-aerosols in healthcare and the dental environment. PLOS ONE. 2017;12(5):e0178007.

https://doi.org/10.1371/journal.pone.0178007 
10. Enwemekaa CE, Bumaha VV, Masson-Meyersc SD. Light as a potential treatment for pandemic coronavirus infections: A Perspective. J Photochem Photobiol

B.
2020;207:111891. doi: 10.1016/j.jphotobiol.2020. 111891

\section{Corresponding author:}

Edwin Sever Bechir

George Emil Palade University of Medicine, Pharmacy, Science, and Technology of Târgu-Mureș, Faculty of Dental Medicine, 38 Gh. Marinescu Str., 540139, Târgu Mureș, Romania

Email: bechir.edwin@gmail.com

Received: July 6, 2020 / Accepted: July 18, 2020 